\title{
Compact Microstrip Bandpass Diplexer Based on Twist Revised Split Ring Resonators
}

\author{
Jian Li, ${ }^{1}$ Yongjun Huang, ${ }^{1}$ Xuefeng Zhao, ${ }^{1,2}$ and Guangjun Wen ${ }^{1}$ \\ ${ }^{1}$ Centre for RFIC and System Technology, School of Communication and Information Engineering, \\ University of Electronic Science and Technology of China, Chengdu 611731, China \\ ${ }^{2}$ School of Computer Engineering, Huaihai Institute of Technology, Lianyungang 222005, China
}

Correspondence should be addressed to Yongjun Huang; yongjunh@uestc.edu.cn

Received 19 February 2015; Accepted 30 March 2015

Academic Editor: Roman E. Noskov

Copyright (c) 2015 Jian Li et al. This is an open access article distributed under the Creative Commons Attribution License, which permits unrestricted use, distribution, and reproduction in any medium, provided the original work is properly cited.

\begin{abstract}
Based on the twist revised split ring resonators (TR-SRRs) inspired filter unit a microstrip bandpass diplexer with highly compact size and high frequency selection and isolation properties is synthesized and systematically characterized. The proposed filter unit exhibits both electric and magnetic coupling effects and possesses two resonance modes (magnetic and electronic resonances). The two resonance modes can be flexibly controlled by adjusting the gap between the two TR-SRRs. The synthesized diplexer has very simple configuration with size of $0.217 \lambda_{d} \times 0.217 \lambda_{d}$ and degree of freedom for impedance matching. Measurement and simulation demonstrations are performed in this paper and a good agreement is achieved. The measured results indicate two quite close frequency channels (centered at $2.16 \mathrm{GHz}$ and $2.91 \mathrm{GHz}$ ) with isolation larger than $30 \mathrm{~dB}$. The proposed diplexer can be easily integrated into miniaturized $\mathrm{RF} /$ microwave integrated circuits.
\end{abstract}

\section{Introduction}

In modern wireless communications, the obvious development tendency is to integrate more than one communication mode in a single termination. Based on this requirement, some multiband/wideband antennas have been designed and therefore we need filters following the antennas to separate the different signals toward different frequency channels and modules $[1,2]$. Various diplexers/triplexes have been reported in recent years based on different filter and/or resonator units, for example, parallel-coupled filter [3], Tshaped resonator [4], stub-loaded resonator [5, 6], quarterwavelength resonator [7], dual-mode resonator [8], and common shorted stubs [9] to realize the assignments. In particular, the filter designs focusing on compact size, high isolation, and out-of-band rejection properties are developed based on stepped impedance coupled-line resonator [10, 11], double-sided parallel-strip line [12], half-wavelength openloop resonators [13], hybrid resonators [14], series LC tanks [15], and substrate integrated waveguide resonator [16].
On the other hand, the artificial structured resonators with dimensional size much smaller than the operating wavelength have been attracting exciting interests in the designs of compact diplexers/triplexes. For example, the split ring resonator (SRR) as the basic artificial unit cell has been widely used to design microwave/millimeter wave components [17] and its complementary inclusion applied to the coplanar waveguide and substrate integrated waveguide can realize high performance diplexers [18, 19]. By using composite quarter-wave right/left-handed (CRLH) resonators or balanced CRLH resonators [20-23], one can obtain more compact size compared with the conventional diplexers/triplexes. However, most of these archives including conventional resonators and artificial structured resonators have complex inclusions, precise-designed T-junction, and impedance circuits which will result in large size and high costs.

In this paper, a new type of diplexer is synthesized by directly using two groups of revised SRR (by electric shorting one end of the SRR to ground plane) pairs with twist arrangements. The proposed twist revised SRR (TR-SRR) inspired 
filter unit exhibits both electric and magnetic coupling effects and has two resonance modes (electric and magnetic resonances) with very small electric size. Therefore the designed diplexer possesses highly compact size compared with other recently reported inclusions. The TR-SRR and filter unit are firstly analyzed, and then the synthesized diplexer is demonstrated by both experimental and numerical methods. Finally the comparisons with previously reported diplexers are presented and discussed.

\section{Filter Design and Analysis}

Figure 1(a) shows the top and side views of the proposed revised SRR configuration. Two extended microstrip lines are collected to the split of a conventional SRR and one of the extended ends is electric shorted to ground plane. Such kind of design can be considered as a new quarterwavelength resonator from the equivalent circuit view. The new revised SRR can exhibit impedance matching to the $50 \Omega$ feed line, which connects to the shorted branch as shown in Figure 1(a), without additional complex circuits. The electric and magnetic fields at the resonance frequency for one revised SRR are shown in Figure 1(b), which indicate asymmetric field distributions and will give us abundant choices for the coupling filter design. After numerical investigations by HFSS full-wave simulations, the twist arrangement as shown in the inset of Figure 1(c) has better passband characteristics [24] and also can be easily used to synthesize the diplexer which will be discussed later. As an example, the simulated transmission and reflection properties of the designed filter unit are shown in Figure 1(c) with parameters shown as follows, in millimeter: $a=4, b=2.7, c=$ $0.4, w=0.4, r=0.15, g=0.5$, and $t=0.8$. Rogers RO4003 substrate, which has the relative dielectric constant $\varepsilon_{r}=3.55$ and loss tangent $\tan \delta=0.0027$, is used in this paper and the thickness of the SRRs and ground plane is $0.03 \mathrm{~mm}$. The dimension of filter unit is $8 \mathrm{~mm} \times 12.4 \mathrm{~mm}$. As can be seen, such filter exhibits a passband centered at $2.97 \mathrm{GHz}$ with a $3 \mathrm{~dB}$ bandwidth of $0.46 \mathrm{GHz}$. From the reflection curve in Figure 1(c) there are two poles at 2.88 and $3.05 \mathrm{GHz}$ and the surface currents on the twist SRR pairs at such two frequencies show typical electric and magnetic resonance modes. Moreover, there is a transmission zero below the passband. From the mixed coupling filter theory, the magnetic coupling for our twist SRR based filter is larger than the electric coupling, and as a result the transmission in lower band is related to strong magnetic coupling $[25,26]$. It can contribute to the high isolation when synthesizing the diplexer in the next section by using the proposed filter unit.

By altering the gap values between the TR-SRR pairs, one can obtain the changing properties of the filter passband as shown in Figure 2(a). It indicates an expanded bandwidth when decreasing the gap. This is due to the enhancements of both electric and magnetic coupling in the adjacent area. Figure 2(b) shows the captured frequency shift properties of the two resonance modes as functions of gap value $g$. The coupling coefficient $k$ and the external quality factors $Q_{e}$ can then be calculated from the two resonance frequencies by using coupling theory [26]:

$$
\begin{aligned}
k & =\frac{\left(f_{2}^{2}-f_{1}^{2}\right)}{\left(f_{2}^{2}+f_{1}^{2}\right)}, \\
Q_{e 1} & =\frac{f_{1}}{\Delta f_{3 \mathrm{~dB}}}, \\
Q_{e 2} & =\frac{f_{2}}{\Delta f_{3 \mathrm{~dB}}},
\end{aligned}
$$

where $f_{1}$ and $f_{2}$ correspond to the lower and higher resonance frequencies and $\Delta f_{3 \mathrm{~dB}}$ is the $3 \mathrm{~dB}$ frequency bandwidth. In our previous work [24], it has shown that our design exhibits high external quality factors, which can contribute to low insertion loss, flat passband, and good return loss. The calculated coupling coefficient shown in Figure 2(b) indicates that when decreasing the gap values the coupling between the two SRRs will be enhanced. However, at smaller gap values, the coupling is too strong so that the two resonance modes separate far from each other and result in the dropdown within the passband as shown in Figure 2(a). Therefore, both the bandwidth and insertion loss will be considered when choosing the gap values, and the isolation will be also taken into consideration when designing the diplexer.

\section{Diplexer Synthesis and Demonstration}

Based on the above analysis for the filter unit, here the diplexer is synthesized by placing properly two filter units side by side with different sizes and connecting to the input port with a simple T-junction as shown in Figure 3(a). Such design can effectively use the limited space to achieve the highly compact inclusion. By using the same Rogers RO4003 substrate as above, the dimensional parameters are finally optimized as follows, in millimeter: $a_{1}=5, a_{2}=4, b_{1}=3.7$, $b_{2}=2.7, c=0.4, e=9.2, f=1.76, g_{1}=0.5, g_{2}=0.5$, $w=0.4$, and $r=0.15$. The overall dimensional size is $d \times d=$ $16 \mathrm{~mm} \times 16 \mathrm{~mm}$. The photograph of fabricated diplexer is also shown in Figure 3(b) with the definitions of the three ports. Each port is soldered with a $50 \Omega$ microminiature coaxial connectors, operated from DC to $6 \mathrm{GHz}$.

Then the scattering parameters for the diplexer are experimentally measured by a two-port vector network analyzer (Agilent N5230A). In measurements, the third port is terminated by a wide-band $50 \Omega$ load when measuring two of these three ports for the diplexer. The measured results are shown in Figure 4 and the corresponding numerical results are also presented for comparisons. As can be seen, good agreements are achieved between measurements and simulations which demonstrate the corrections of the design and synthesis. The measured two channels are located at $2.16 \mathrm{GHz}$ and $2.91 \mathrm{GHz}$ with $3 \mathrm{~dB}$ bandwidths of $0.3 \mathrm{GHz}$ and $0.32 \mathrm{GHz}$, respectively. The measured insertion losses are $1.25 \mathrm{~dB}$ and $1.48 \mathrm{~dB}$, respectively, for the two channels, which show quite low values due to the simple configuration (including the extra losses from the microminiature coaxial 


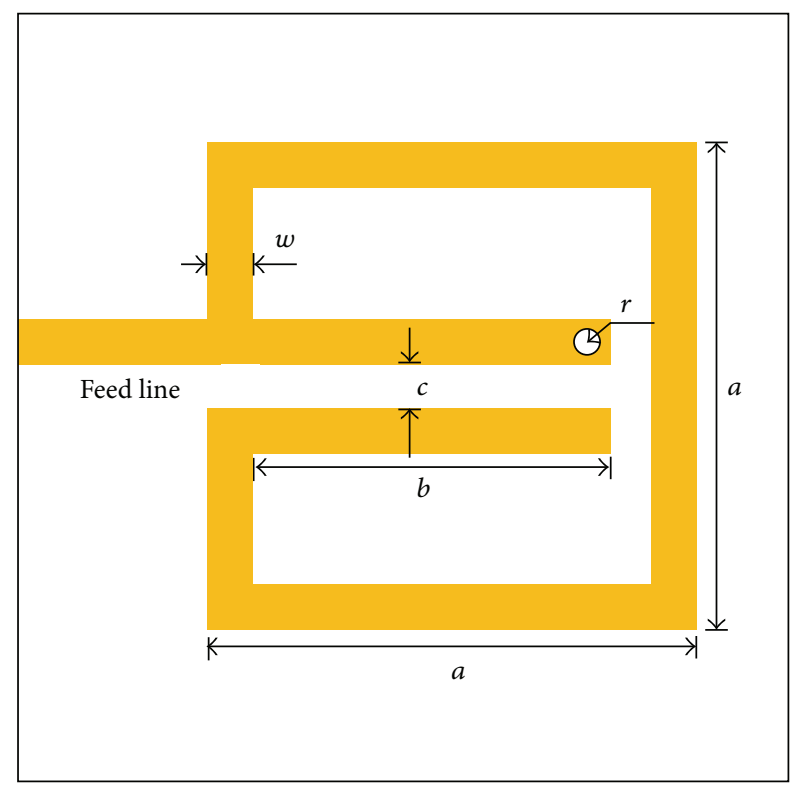

Top view

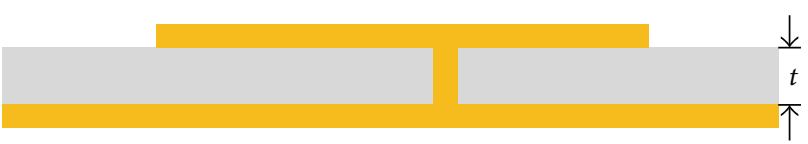

Side view

(a)

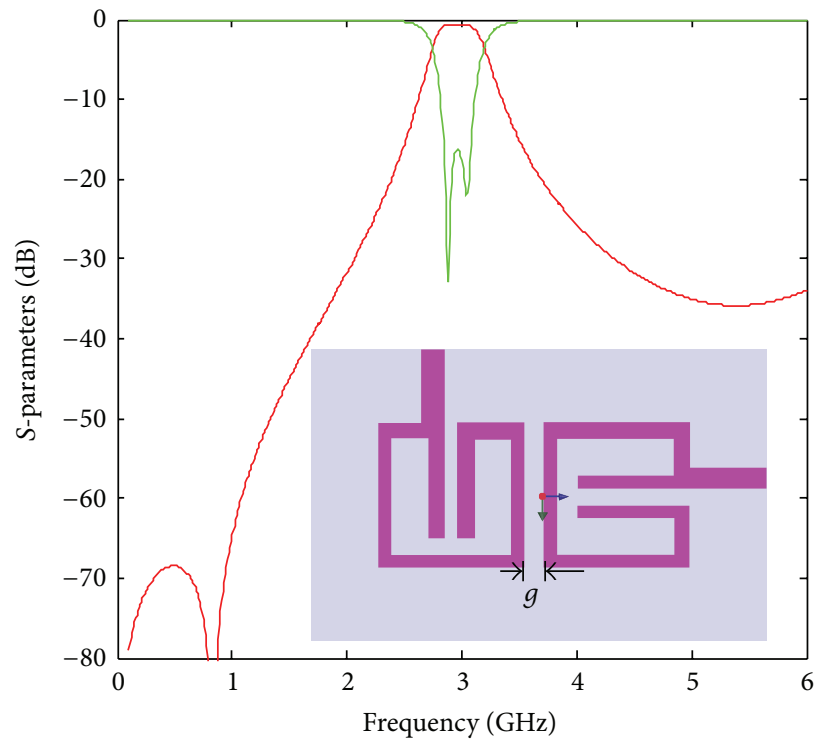

$-\left|S_{11}\right|$

$-\left|S_{21}\right|$

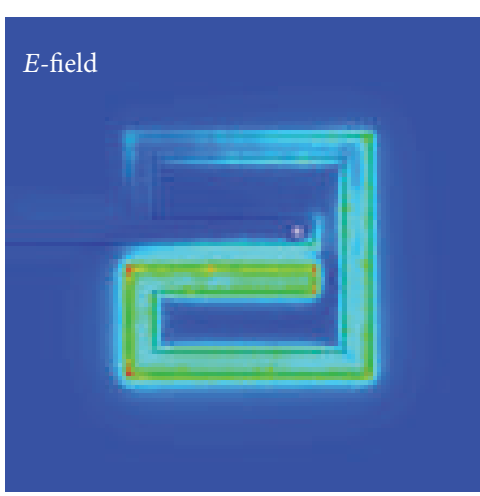

\section{$H$-field}

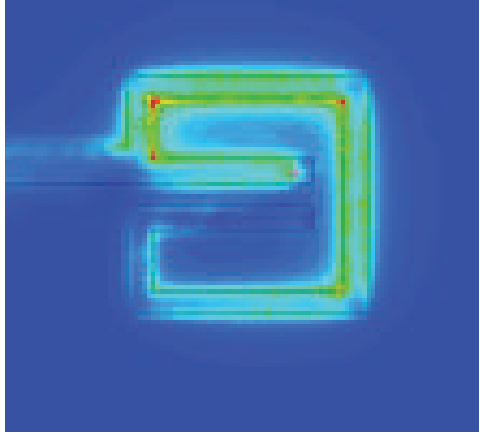

(b)
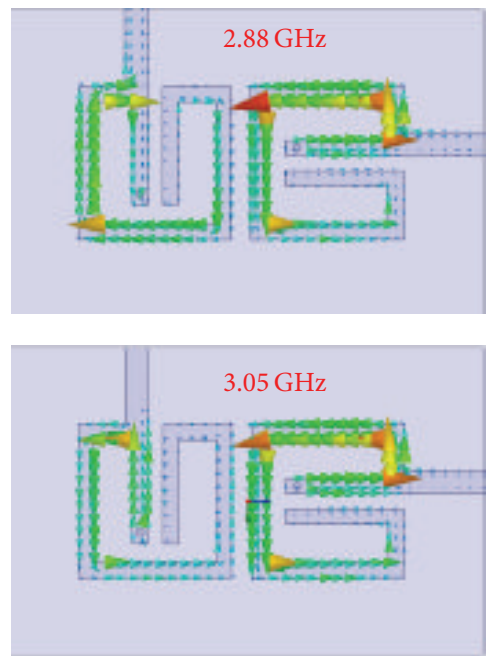

(d)

FIGURE 1: (a) The new revised SRR, (b) the electric and magnetic field distributions near the SRR at resonance frequency, (c) the twist SRRs inspired filter unit and its simulated scattering parameters, and $(\mathrm{d})$ the surface current distributions at the two resonance modes. 


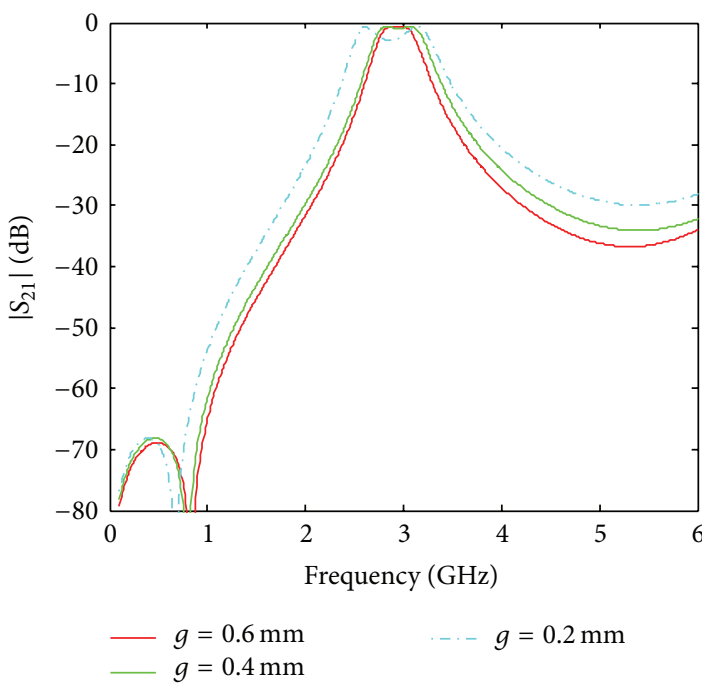

(a)

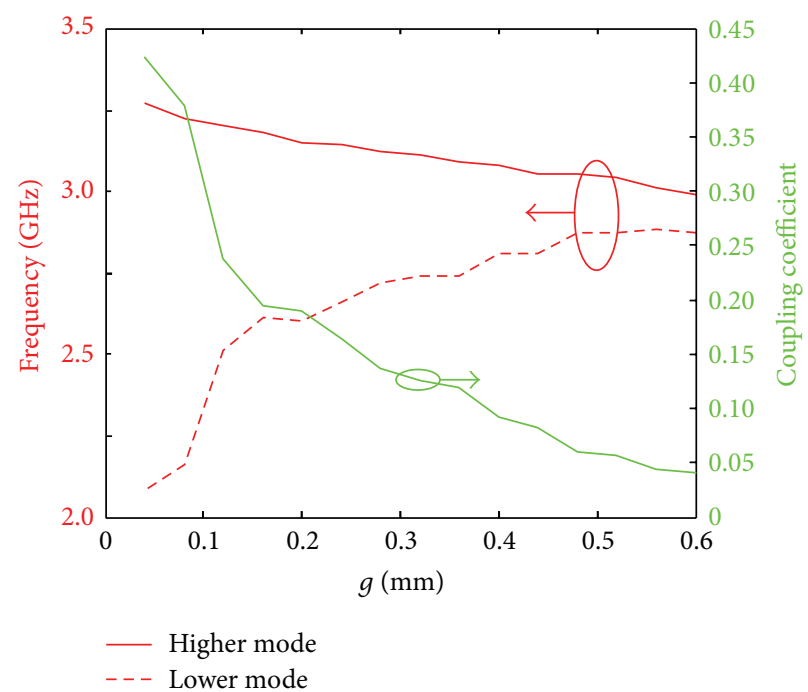

(b)

FIgURE 2: (a) Simulated transmissions for the twist SRR inspired filter unit at different gaps and (b) the changing properties for the two resonance frequencies and the calculated mix coupling coefficient as functions of gap $g$.

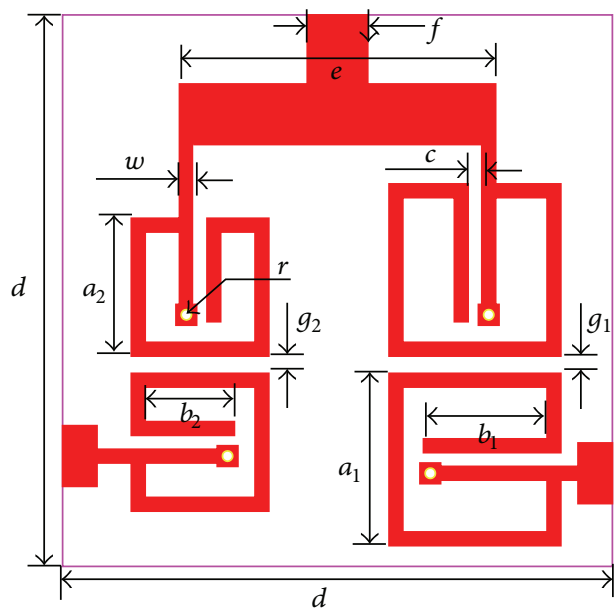

(a)

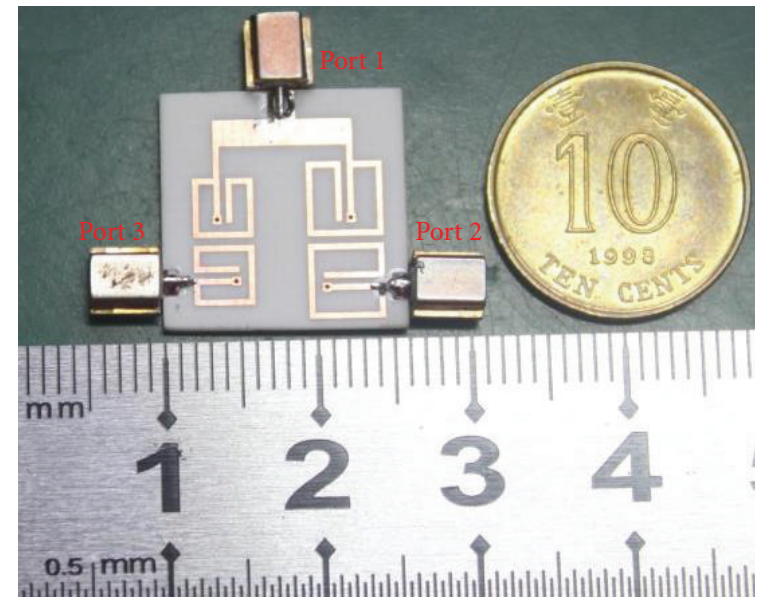

(b)

FIgURE 3: (a) The synthesized compact diplexer layout with dimensional parameters and (b) the photograph of fabricated diplexer.

connectors). The return losses for the input port (Port 1) at the two channels are $25 \mathrm{~dB}$ and $14 \mathrm{~dB}$ and for the two output ports (Ports 2 and 3 ) are $26 \mathrm{~dB}$ and $21 \mathrm{~dB}$. These are quite good impedance matching properties even though they are without a carefully designed matching circuit (just conventional $50 \Omega$ microstrip line). The out-of-band rejection is more than $30 \mathrm{~dB}$ and reaches up to $5 \mathrm{GHz}$ for the lower channel and in the entire measured frequency region for the higher channel. Moreover, the measured isolation between the two output ports shows a value of larger than $30 \mathrm{~dB}$, which is also a good isolation property for the proposed highly compact inclusion. Such isolation can be further increased by adding more SRRs in the filter units to get more transmission zeros below and above the operating bands but will destroy the compact configuration size.

Finally, the performance comparisons for the proposed diplexer and other recently reported designs are concluded in Table 1 . Here $\lambda_{d}$ is the operating wavelength within the dielectric substrate defined as $\lambda_{d}=c /\left(\sqrt{\varepsilon_{r}} f\right)$, where $\varepsilon_{r}$ is the relative permittivity of substrate, $f$ is the center frequency of first channel, and $c$ is the speed of light in free space. It can be found that the diplexer proposed in this paper has comparable frequency selection and isolation properties and almost the smallest inclusion size (except the design reported in [13] using complex series LC tanks and meander line inductors to achieve the compact resonators). 


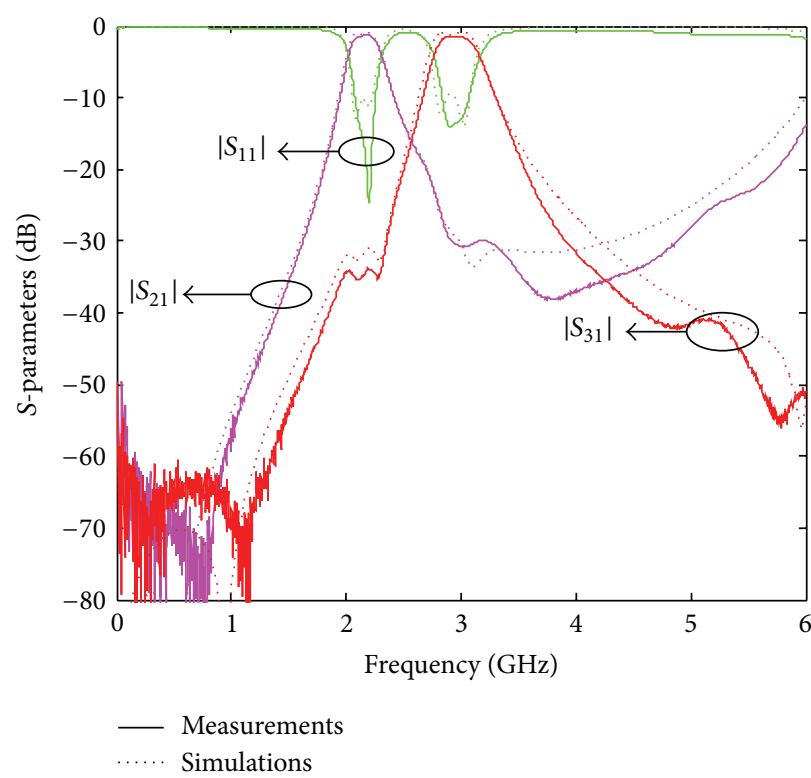

(a)

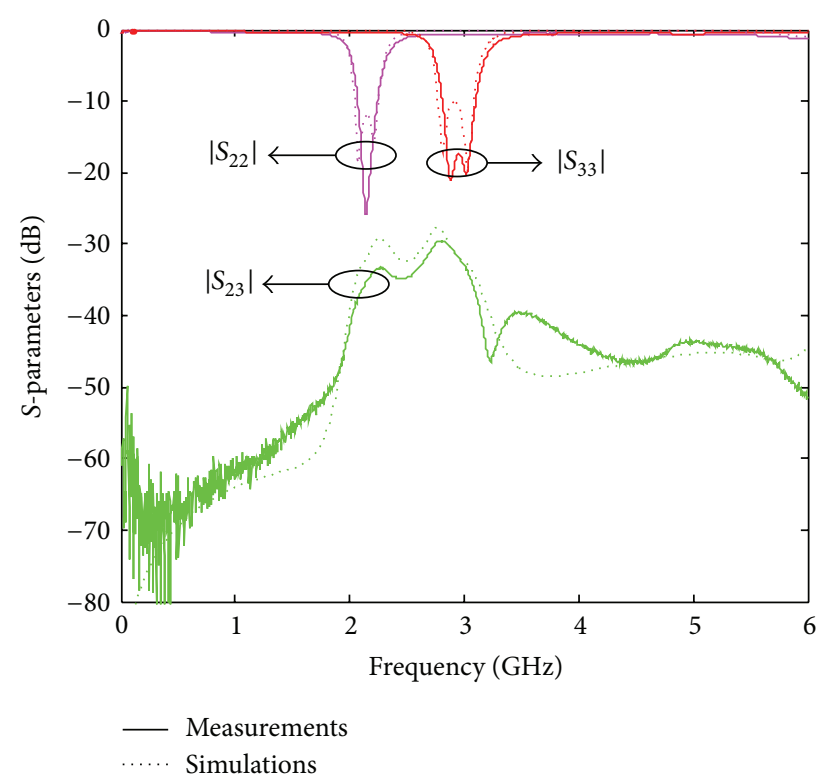

(b)

Figure 4: (a) The measured and simulated reflections of Port 1 and transmissions of Ports 2 and 3 from Port 1 and (b) reflection for Ports 2 and 3 and the transmission between Ports 2 and 3.

TABLE 1: Comparisons between the proposed diplexer and other compact designs.

\begin{tabular}{lccccc}
\hline Designs & $\varepsilon_{r}$ of substrate & Frequency $(\mathrm{GHz})$ & Size $\left(\lambda_{d}{ }^{2}\right)$ & Insertion loss $(\mathrm{dB})$ & Isolation $(\mathrm{dB})$ \\
\hline$[11]$ & 4.5 & $1.85,2.5$ & $0.424 \times 0.382$ & $2.05,2.15$ & $>2.51,2.17$ \\
{$[14]$} & 2.2 & $1.82,2.5$ & $0.193 \times 0.297$ & $0.4,0.5$ & $>55$ \\
{$[15]$} & 3.38 & $1.8,3.6$ & $0.153 \times 0.09$ & $2.86,3.04$ & $>30$ \\
{$[16]$} & 2.55 & 1.9 & $1.325 \times 1.325$ & $1.34,1.44$ & $>40$ \\
{$[22]$} & 2.2 & $3.5,2.35$ & $0.178 \times 0.341$ & $1.95,1.97$ & $>25$ \\
{$[23]$} & 2.65 & $2.16,2.91$ & $0.944 \times 0.765$ & $1.25,1.48$ & $>25$ \\
This work & 3.55 & $0.217 \times 0.217$ & 330 \\
\hline
\end{tabular}

\section{Conclusions}

In this paper, a new type of TR-SRRs inspired bandpass filter unit is designed and then a highly compact diplexer based on the designed filters is synthesized and demonstrated. Such diplexer with size of $0.217 \lambda_{d} \times 0.217 \lambda_{d}$ exhibits good characteristics, including the close channels, low insertion loss, wide out-of-band rejection, and high isolation, which can be flexibly integrated into miniaturized RF/microwave/millimeter wave circuits and systems.

\section{Conflict of Interests}

The authors declare that there is no conflict of interests regarding the publication of this paper.

\section{Acknowledgments}

This work was supported in part by the National Natural Science Foundation of China (Grant no. 61371047), Research Fund for the Doctoral Program of Higher Education of China (Grant no. 20110185110014), and the Fundamental Research
Funds for the Central Universities (Grant no. E022050205). Yongjun Huang also gratefully acknowledges the Scholarship Award for Excellent Doctoral Student granted by Ministry of Education of China (Grant no. A03003023901006).

\section{References}

[1] B. Strassner and K. Chang, "Wide-band low-loss high-isolation microstrip periodic-stub diplexer for multiple-frequency applications," IEEE Transactions on Microwave Theory and Techniques, vol. 49, no. 10, pp. 1818-1820, 2001.

[2] Z. C. Hao, W. Hong, J. X. Chen, X. P. Chen, and K. Wu, "Planar diplexer for microwave integrated circuits," IEE ProceedingsMicrowaves, Antennas and Propagation, vol. 152, no. 6, pp. 455459, 2005.

[3] Y.-S. Lin, P.-C. Wang, C.-W. You, and P.-Y. Chang, "New designs of bandpass diplexer and switchplexer based on parallelcoupled bandpass filters," IEEE Transactions on Microwave Theory and Techniques, vol. 58, no. 12, pp. 3417-3426, 2010.

[4] M.-L. Chuang and M.-T. Wu, "Microstrip diplexer design using common T-shaped resonator," IEEE Microwave and Wireless Components Letters, vol. 21, no. 11, pp. 583-585, 2011. 
[5] Q. Xue, J. Shi, and J.-X. Chen, "Unbalanced-to-balanced and balanced-to-unbalanced diplexer with high selectivity and common-mode suppression," IEEE Transactions on Microwave Theory and Techniques, vol. 59, no. 11, pp. 2848-2855, 2011.

[6] C. F. Chen, C. Y. Lin, B. H. Tseng, and S. F. Chang, "Highisolation and high-rejection microstrip diplexer with independently controllable transmission zeros," IEEE Microwave and Wireless Components Letters, vol. 24, no. 12, pp. 851-853, 2014.

[7] F. Cheng, X. Q. Lin, Z. B. Zhu, L. Y. Wang, and Y. Fan, "High isolation diplexer using quarter-wavelength resonator filter," Electronics Letters, vol. 48, no. 6, pp. 330-331, 2012.

[8] B. A. Belyaev, A. M. Serzhantov, and V. V. Tyurnev, "A microstrip diplexer based on dual-mode resonators," Technical Physics Letters, vol. 38, no. 8, pp. 743-746, 2012.

[9] W. Che, X. Gao, and W. Feng, "Microstrip diplexer for GSM and WLAN bands using common shorted stubs," Electronics Letters, vol. 50, no. 20, pp. 1486-1488, 2014.

[10] S. Srisathit, S. Patisang, R. Phromloungsri, S. Bunnjaweht, S. Kosulvit, and M. Chongcheawchamnan, "High isolation and compact size microstrip hairpin diplexer," IEEE Microwave and Wireless Components Letters, vol. 15, no. 2, pp. 101-103, 2005.

[11] H. Liu, W. Xu, Z. Zhang, and X. Guan, "Compact diplexer using slotline stepped impedance resonator," IEEE Microwave and Wireless Components Letters, vol. 23, no. 2, pp. 75-77, 2013.

[12] Q. Xue and J.-X. Chen, "Compact diplexer based on doublesided parallel-strip line," Electronics Letters, vol. 44, no. 2, pp. 123-124, 2008.

[13] Z.-H. Bao, J.-X. Chen, E. H. Lim, and Q. Xue, "Compact microstrip diplexer with differential outputs," Electronics Letters, vol. 46, no. 11, pp. 766-768, 2010.

[14] T. Yang, P.-L. Chi, and T. Itoh, "High isolation and compact diplexer using the hybrid resonators," IEEE Microwave and Wireless Components Letters, vol. 20, no. 10, pp. 551-553, 2010.

[15] J.-Y. Zou, C.-H. Wu, and T.-G. Ma, "Miniaturized diplexer using synthesized microstrip lines with series LC tanks," IEEE Microwave and Wireless Components Letters, vol. 22, no. 7, pp. 354-356, 2012.

[16] F. Cheng, X. Lin, K. Song, Y. Jiang, and Y. Fan, "Compact diplexer with high isolation using the dual-mode substrate integrated waveguide resonator," IEEE Microwave and Wireless Components Letters, vol. 23, no. 9, pp. 459-461, 2013.

[17] R. Marqués, F. Martín, and M. Sorolla, Metamaterials with Negative Parameters: Theory, Design and Microwave Applications, John Wiley \& Sons, Hoboken, NJ, USA, 2007.

[18] J. Bonache, I. Gil, J. García-García, and F. Martín, "Complementary split ring resonators for microstrip diplexer design," Electronics Letters, vol. 41, no. 14, pp. 810-811, 2005.

[19] Y. Dong and T. Itoh, "Substrate integrated waveguide loaded by complementary split-ring resonators for miniaturized diplexer design," IEEE Microwave and Wireless Components Letters, vol. 21, no. 1, pp. 10-12, 2011.

[20] Y. Horii, C. Caloz, and T. Itoh, "Super-compact multilayered left-handed transmission line and diplexer application," IEEE Transactions on Microwave Theory and Techniques, vol. 53, no. 4, pp. 1527-1533, 2005.

[21] T. Kodera and C. Caloz, "Integrated leaky-wave antennaduplexer/diplexer using CRLH uniform ferrite-loaded open waveguide," IEEE Transactions on Antennas and Propagation, vol. 58, no. 8, pp. 2508-2514, 2010.

[22] T. Yang, P.-L. Chi, and T. Itoh, "Compact quarter-wave resonator and its applications to miniaturized diplexer and triplexer," IEEE
Transactions on Microwave Theory and Techniques, vol. 59, no. 2, pp. 260-269, 2011.

[23] H. Zeng, G. Wang, D. Wei, and Y. Wang, "Planar diplexer using composite right-/left-handed transmission line under balanced condition," Electronics Letters, vol. 48, no. 2, pp. 104-106, 2012.

[24] Y. Huang, G. Wen, and J. Li, "Systematical analysis for the mixed couplings of two adjacent modified split ring resonators and the application to compact microstrip bandpass filters," AIP Advances, vol. 4, no. 10, Article ID 107119, 2014.

[25] B. Wu, X. Lai, T. Su, and C.-H. Liang, "Wideband cross coupled filter using split-ring resonator defected ground structure," Journal of Electromagnetic Waves and Applications, vol. 22, no. 11-12, pp. 1631-1638, 2008.

[26] J. S. Hong and M. J. Lancaster, Microstrip Filters for RF Microwave Application, chapter 8, John Wiley \& Sons, New York, NY, USA, 2001. 

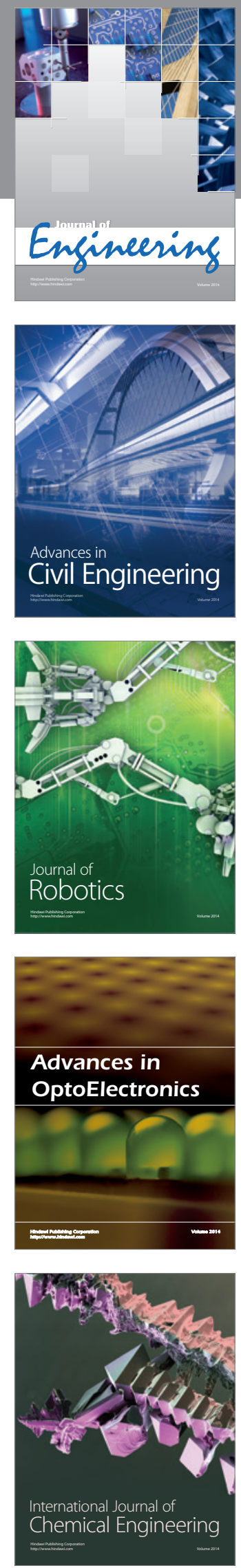

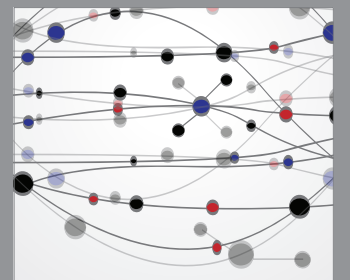

The Scientific World Journal
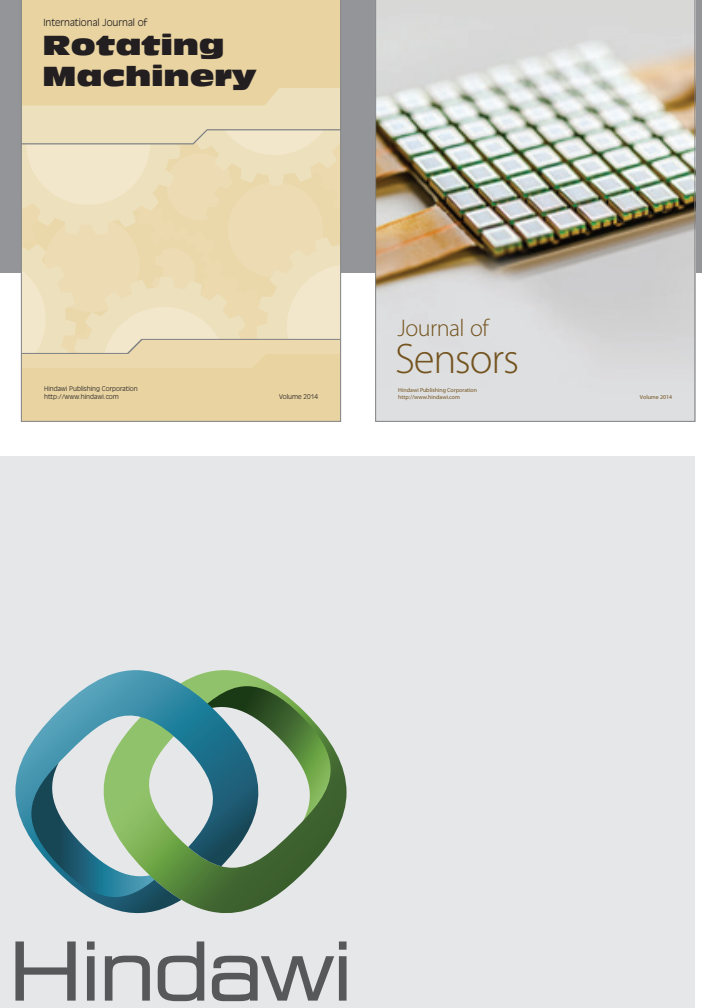

Submit your manuscripts at http://www.hindawi.com
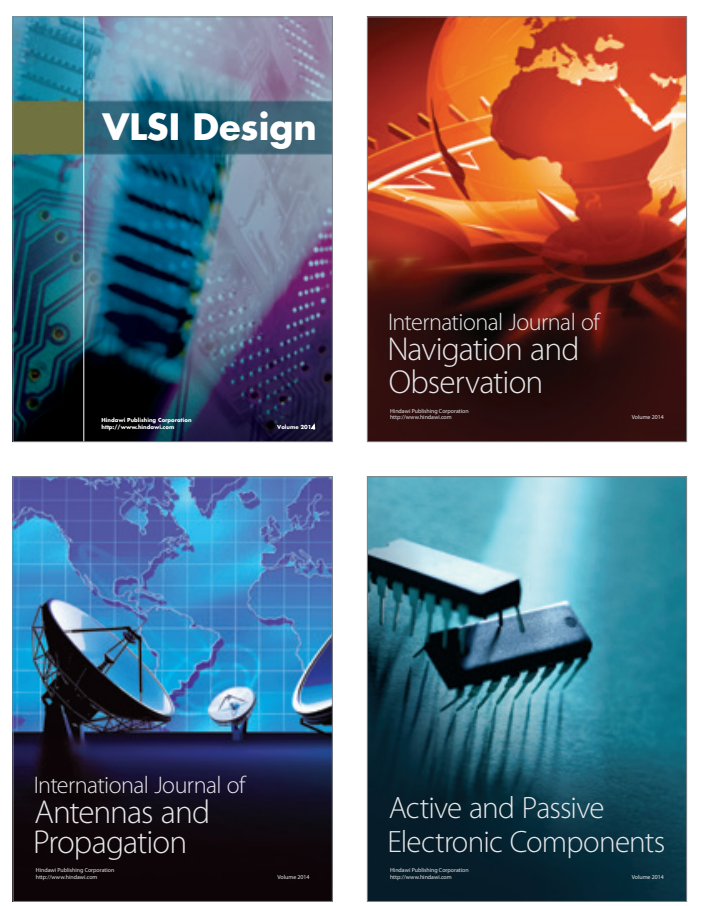
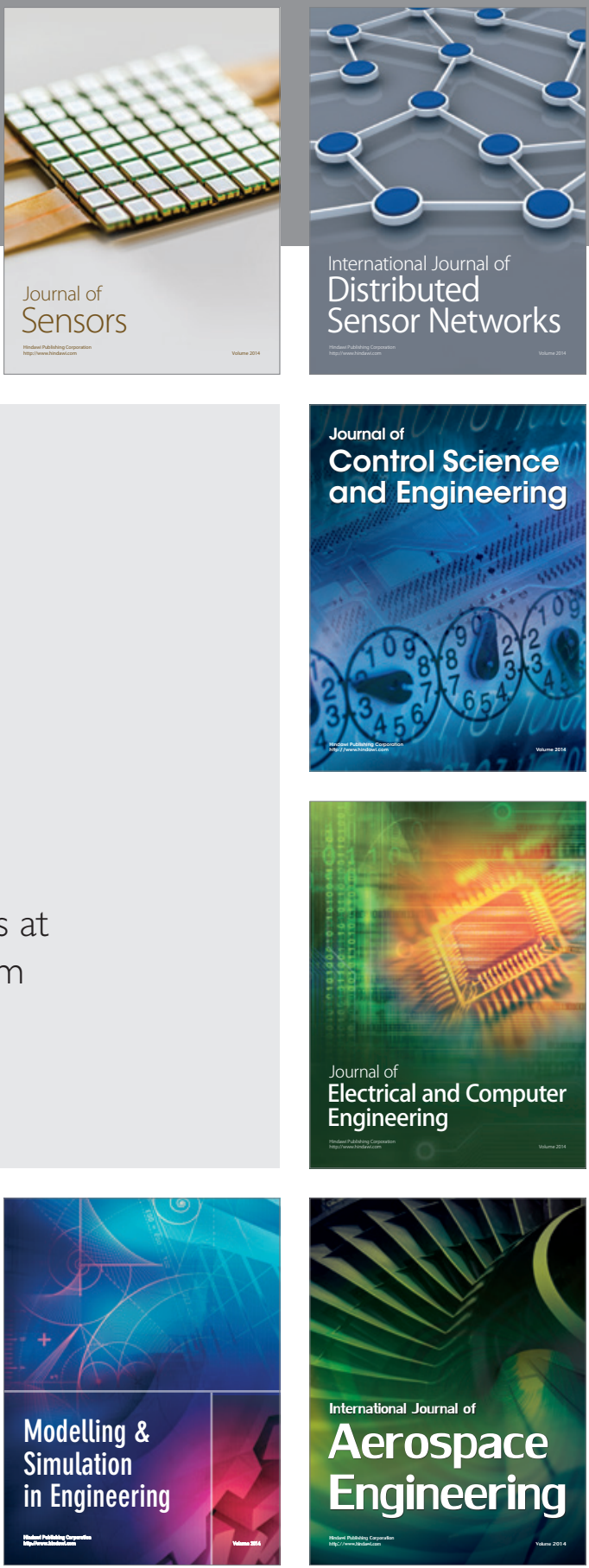

Journal of

Control Science

and Engineering
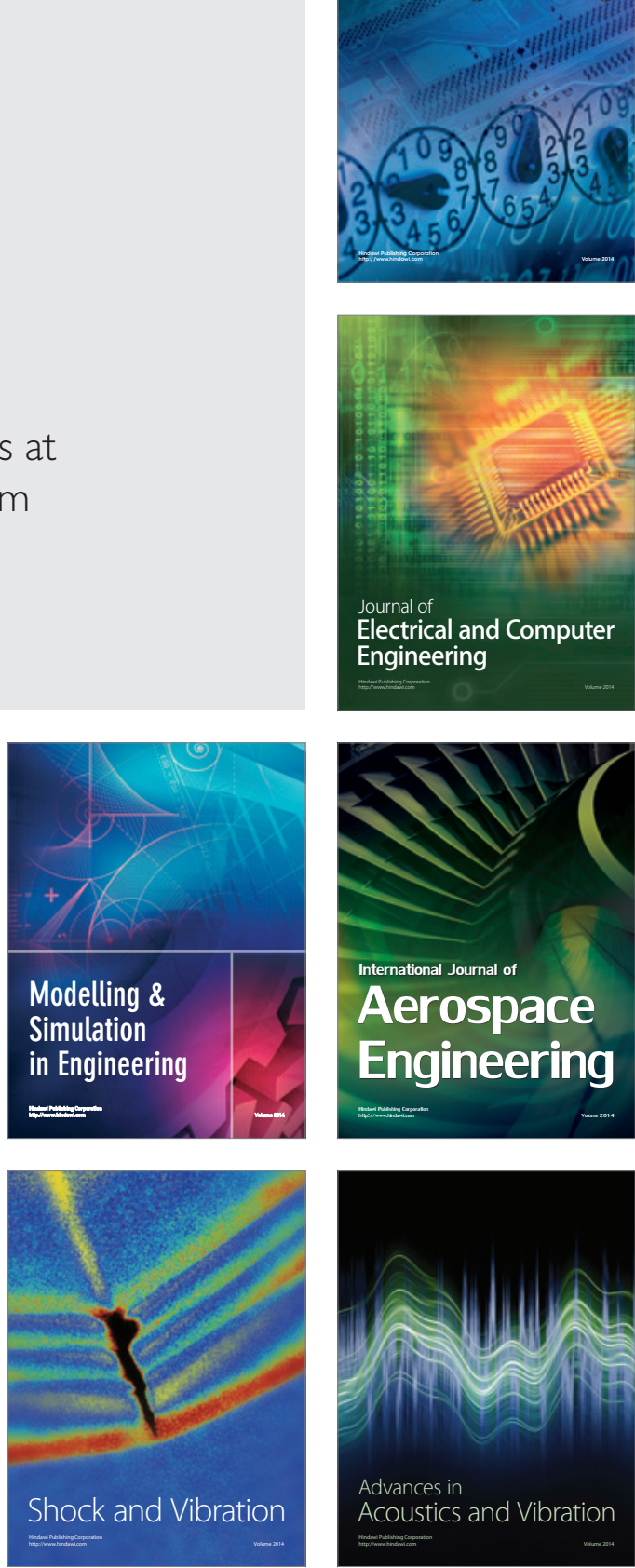